failure of antigen production is more likely to be due to genetic change associated with incomplete penetrance than to genetic loss. An alternative or additional possibility cannot, however, be excluded-that exuberant cell division causes dilution of a particulate cytoplasmic factor responsible for the formation of the antigen.

Loss of organ-specific antigens could influence tumour metastasis by diminishing normal cell adhesion at the primary tumour site or by removing specific cell differences which might prevent secondary growth of tumour cells in another organ. A preliminary study has been made of the possible importance of the gastrointestinal-specific antigen in this connexion: it could, in theory, behave as a "marker" antigen by coating the mucosal cell surface. However, the information so far available is too meagre for any useful discussion, though the absence of antigen in lymph-node metastases of all ten cases examined is a clear indication for further study. More investigation is also required to assess the possible diagnostic usefulness of immunofluorescence staining of gastro-intestinal tumours, especially for distinguishing between benign and premalignant polyps. Long-term follow-up studies of a large series of cases will be needed to confirm that the observed qualitative staining differences provide a reliable means of distinguishing benign from malignant states.

\section{Summary}

A gastro-intestinal-specific antigen demonstrated in mucoid cells by immunofluorescence staining and serological methods, probably an acid mucopolysaccharide, was totally absent from 45 out of 54 gastro-intestinal carcinomas. In the remaining nine carcinomas, five of which were mucoid, staining of antigen was obtained in a small proportion of cells scattered among unstained acini. The immunohistological appearance of four premalignant polyps was similar but the scattered fluorescent cells were more numerous. In eight benign polyps, however, staining was fairly uniform in all cells of the acini, and, though dimmer, showed much the same distribution as in normal mucosa. Lymph-node metastases from ten cases did not stain.

The staining differences, which appear to portray an intrinsic distinction between benign and malignant states, may prove to be of diagnostic value.

This research programme has been supported by grants from the Scottish Hospital Endowments Research Trust and, in the later stages, from the Medical Research Council. We are indebted to our surgical colleagues at Aberdeen Royal Infirmary and Woodend General Hospital for providing surgical specimens, and to Dr. C. M. M. Begg for advice about the possible cytogenetic implications of the antigen loss.

REFERENCES

Burnet, M. (1957). Brit. med. J., 1, 779.

Glynn, L. E., and Holborow, E. J. (1959). Brit. med. Bull., 15, 150.

Goudie, R. B., and McCallum, H. M. (1962). Lancet, 1, 348.

Green, H. N. (1959). In Ciba Foundation Symposium on Carcinogenesis: Mechanisms of Action, p. 131. London. (1961). Nature (Lond.), 192, 1201

Nairn, R. C. (1962a). In Fluorescent Protein Tracing, p. 229 Livingstone, Edinburgh.

(1962b). Ibid. p. 198 .

- Fothergill, J. E., McEntegart, M. G., and Porteous, I. B. (1962). Brit. med. J., 1, 1788 .

McEntegart, M. G., Fothergill, J. E., and Porteous, I. B. (1961). Lancet, 2, 109

Richmond, H. G., McEntegart, M. G., and Fothergill, J. E. (1960) Brit. med. J., 2, 1335

Pikovski, M. A., and Witz, I. P. (1961). Brit. J. Cancer, 15, 584.

Vogt, P. (1960). Z. Naturf., 15b, 221.

Weiler, E (1952), Ibid., 7b, 324 .

(1956a). Ibid., 11b, 31 .

(1956b). Brit. J. Cancer, 10, 553.

(1956c). Ibid., 10, 560.

(1959). In Ciba Foundation Symposium on Carcinogenesis:

Mechanisms of Action, p. 165. London.

\title{
LUMBAR SPINE DENSITOMETRY
}

\section{BY}

\section{B. E. C. NORDIN, M.D., Ph.D., M.R.C.P. Senior Lecturer}

\section{E. BARNETT, L.R.C.P.\&S.Ed., F.F.R., D.M.R.D. Consultant Radiologist}

J. MacGREGOR, M.Sc.

Senior Scientific Assistant

AND

J. NISBET, B.Sc.

Research Assistant

From the Department of Radiology and the University Department of Medicine, Gardiner Institute, Western Infirmary, Glasgow

Spinal osteoporosis is characterized by a loss of trabecular bone which in turn causes a reduction in radiographic bone density. In severe cases the diagnosis is made easily by the subjective assessment of a radiologist, but in milder cases that is quite unreliable. There is therefore a need for a reliable and sensitive method of measuring the density of the vertebral bodies. The available methods applicable to the ulna (Keane, Spiegler, and Davis, 1959 ; Doyle, 1961), the metacarpal (Bywaters, 1948; Koch and Kaplan, 1961), and the os calcis (Mayo, 1961) cannot be applied to the spine, which is mainly affected by the osteoporotic process.

The present paper describes a method of assessing spinal density and presents some preliminary results.

\section{Methods}

The procedure depends on the comparison of the radiographic densities of the vertebral bodies and the intervertebral disks on a lateral tomogram of the lumbar spine. Variations in physical characteristics between exposures, such as line voltage, development, and film speed, are controlled by exposing a specimen of normal human lumbar spine simultaneously. The body:disk density ratio of the patient's spine can therefore be compared with that of the standard spine on the same film.

A dissection of the second, third, and fourth lumbar vertebrae from a normal human spine mounted in formol-saline in a "perspex" box is used as the standard. The depth of the fluid in the box is such that the $x$-ray density of the standard is similar to that of a normal subject of average size.

The patient is placed on the couch in the right lateral position with the standard spine placed against his back. The height of the standard is adjusted with strips of wood until it is in the same horizontal plane as the patient's spine (Fig. 1). A single tomographic cut is then made through the sagittal plane of the patient and the standard. A typical film is shown in Fig. 2.

The images of the spine and the standard are cut out to make two film strips about 2 in. $(5 \mathrm{~cm}$.) wide and 
about 8 in. $(20 \mathrm{~cm}$.) long, which are then fed through a Laurence-Locarte automatic recording densitometer with a $1-\mathrm{mm}$. slit. Both images are of course traced at the same densitometric setting.

The relative vertebral density (R.V.D.) is calculated by subtracting the average density of the patient's vertebral bodies from the average density of the disks, and dividing this numerator by the corresponding difference in the standard. If the patient's vertebral bodies are less dense than the disks (which may occur in osteoporosis) then the numerator, and therefore the R.V.D., ratio will have a negative value. Positive R.V.D. values less than unity signify that the patient's vertebral bodies are denser than the disks in absolute terms, but less dense, relative to the disks, than are those of the standard.

An example of the procedure is shown in Fig. 3, which is a typical tracing of a patient (above) and the standard (below). It will be seen that the disks, bodies, and end-plates are easily identified. The peaks of three good disk tracings are joined by straight lines. A parallel line or lines is then drawn through the plateaus of the tracings of the intervening two bodies, ignoring spikes due to nutrient arteries and minor irregularities.

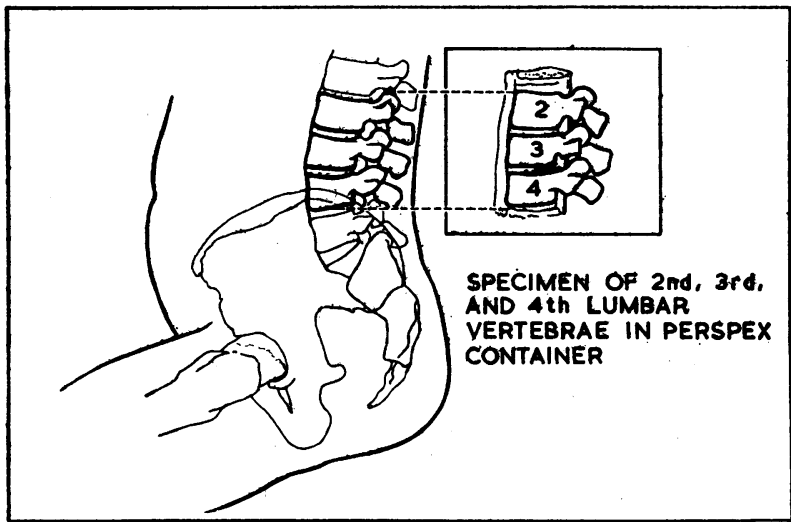

FIG. 1.-Diagrammatic representation of patient and standard positioned for radiography.

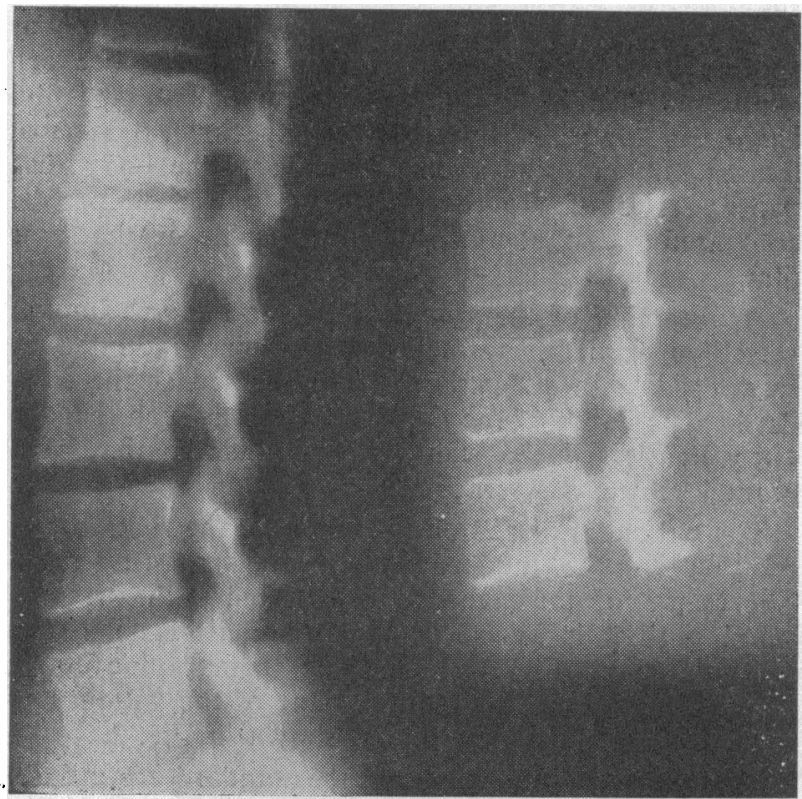

Fio. 2.-An example of a tomogram of the lumbar spine of a patient (left) and the standard.
The distance between these parallel lines represents the difference between densities of the disks and the bodies. In Fig. 3 the average difference in density in the normal subject's spine (in arbitrary units) is $0.3+0.2 \div 2$, or 0.25 unit. The average difference in the standard is 0.18 unit. The R.V.D. is therefore $0.25 \div 0.18$, or +1.4 . In other words, the difference between disk and body density is greater than in the standard, as can be appreciated by simple inspection of the tracings.

However, as shown in Fig. 3, the difference between the soft-tissue thickness at waist-level and the regions immediately above and below produces an overall slope in the tracing which sometimes makes the determination of R.V.D. difficult. Despite this problem, which we are trying to eliminate, the agreement between the R.V.D. values calculated independently by two observers is reasonably satisfactory (Fig. 4).

Results

Fig. 5 shows the relationship between the R.V.D. and the $x$-ray score determined by the method of Barnett and Nordin (1960) in 79 consecutive cases. With the latter method a "total score" of 168 or less is always abnormal, but a higher score does not necessarily denote normal bones (Barnett and Nordin, 1961). As might be expected, therefore, Fig. 5 shows that all cases with total scores of 168 or less have very low R.V.D. values (below 0.4 ), but that many cases with higher total scores also have R.V.D. values of less than unity-that is, vertebral bodies which are relatively less dense than the standard.

The relation between the R.V.D. and the " peripheral score " of Barnett and Nordin (1960) is shown in Fig. 6. With one exception, all cases with peripheral scores of 88 or less (peripheral osteoporosis) have very low R.V.D. values. However, there are many cases in which the
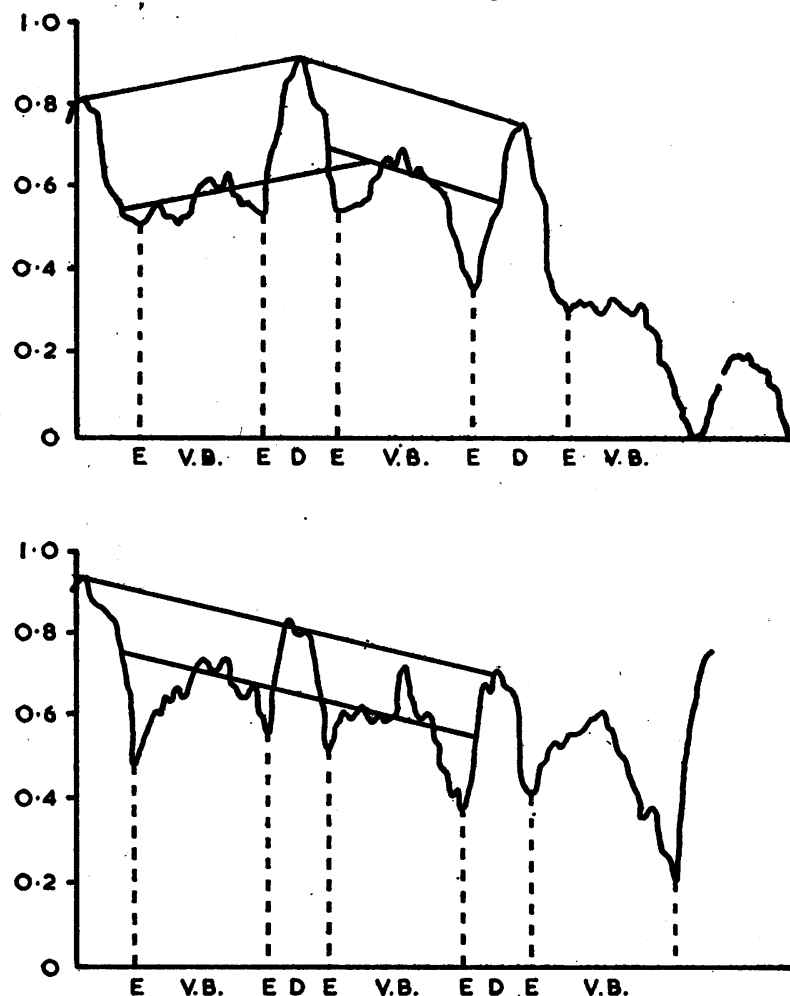

Fig. 3.-Densitometry tracings of a normal lumbar spine (above) and of the standard. The lines have been drawn in to permit calculation of relative density (see text). V.B. $=$ Vertebral body. $\mathrm{B}=$ End-plate. $\mathrm{D}=\mathrm{Disk}$ 
R.V.D. is low, although the peripheral score is normal. Thus peripheral bone osteoporosis is always associated with a reduction in spinal density, but reduced vertebral density may exist in the absence of peripheral osteoporosis.

Fig. 7 shows the relationship between R.V.D. and the degree of spinal biconcavity as estimated by the " spinal score " of Barnett and Nordin (1960), in which a value of less than 81 indicates biconcavity. There is clearly a correlation between these two values, and all but four of the cases with abnormal spinal scores have low R.V.D. values ( 0.6 or less). There are many cases in which the R.V.D. is less than unity, although the spinal score is normal, but in only two of these is the R.V.D. value negative. This suggests that vertebral biconcavity denotes severe osteoporosis, and that reduced density can be present without detectable biconcavity.

\section{Discussion}

The critical feature of the densitometric procedure we have described is the comparison of vertebral body

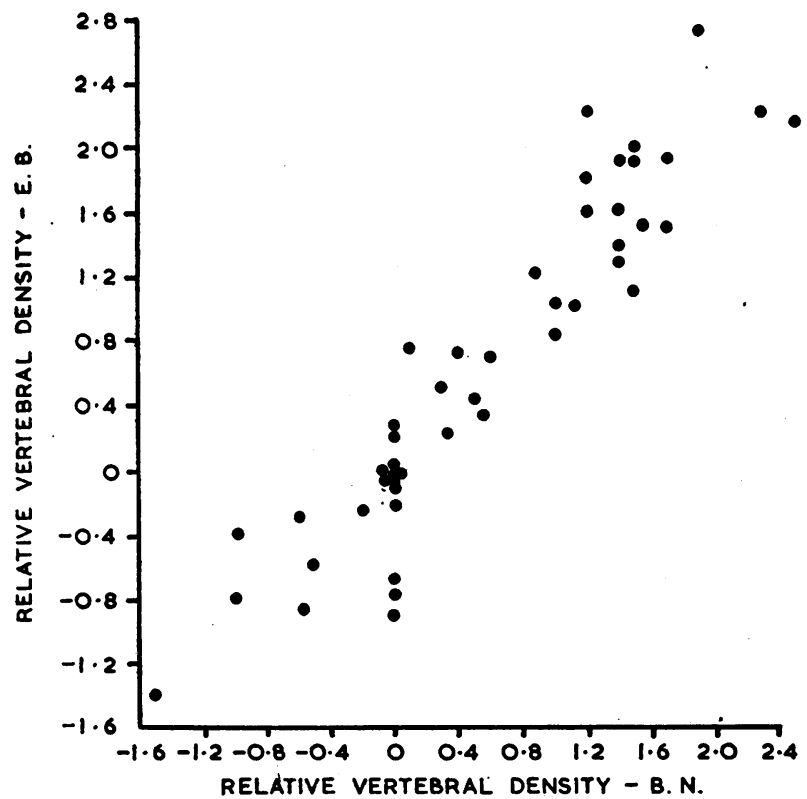

F1G. 4. -Relation between R.V.D. ratios calculated independently from densitometry tracings by two observers.

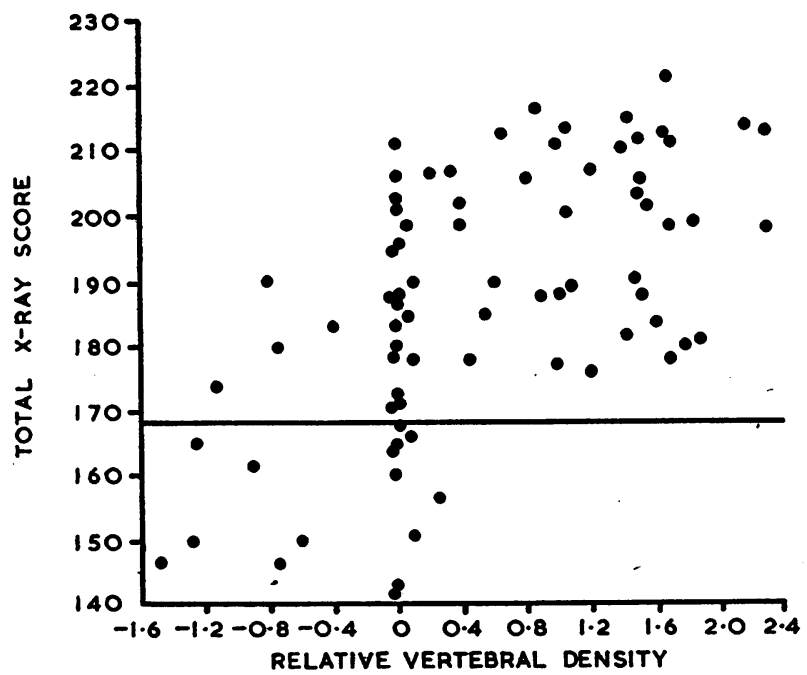

Fio. 5.-Relation between R.V.D. and total $x$-ray score in 79 cases. density with disk density. The relation between these two should not be greatly influenced by variations in soft-tissue mass, in film exposure or development, or by other extrinsic factors. The validity of the method depends upon the assumption that radiological disk density is unaffected by the disease process.

The calculation of relative vertebral density from the densitometry tracing appears to be reasonably reproducible in the hands of experienced observers, although it must be admitted that the tracings vary greatly in quality and must occasionally be discarded.

We have not yet attempted to define the normal range of the R.V.D., but we are collecting control material

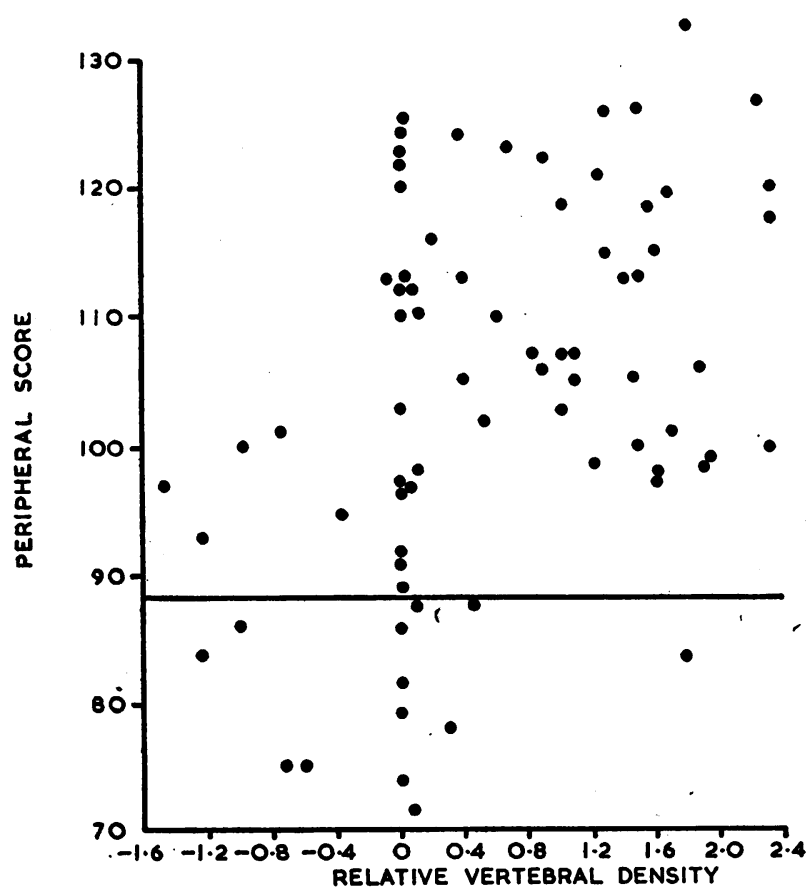

Fig. 6.-Relation between R.V.D. and peripheral $x$-ray score in

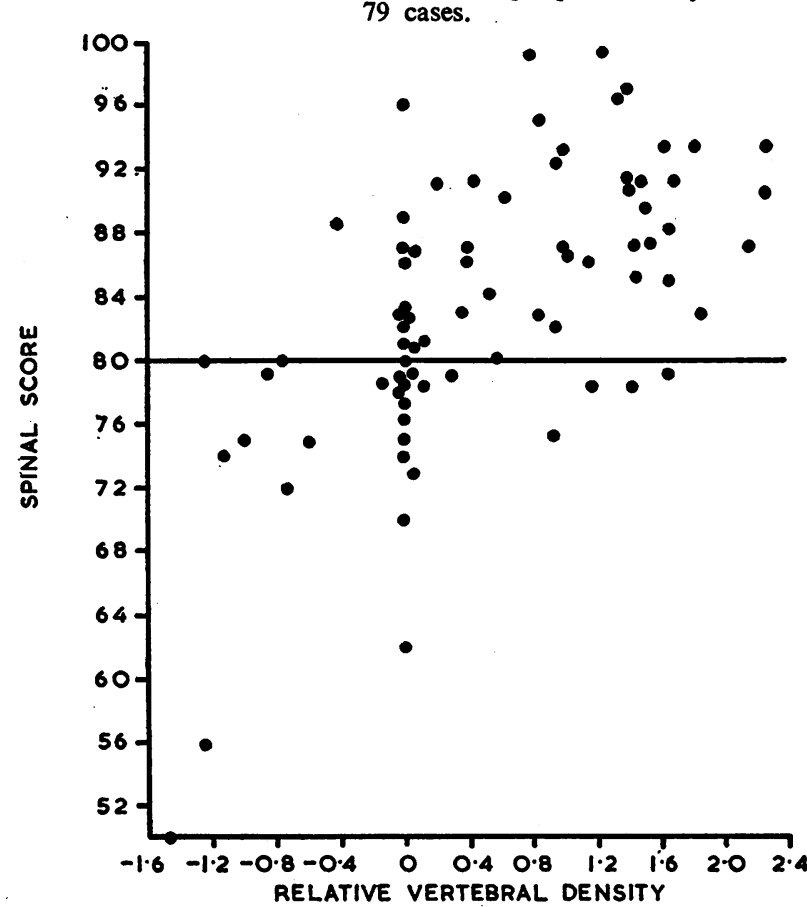

Fig. 7.-Relation between R.V.D. and spinal $x$-ray score in 79 cases. 
which in due course will enable us to define the distribution in statistical terms. In the meantime an R.V.D. ratio of less than unity indicates a vertebral density less than our standard. We are not yet prepared to define the reduction in bone density which justifies the use of the term " osteoporosis," but consider it axiomatic that vertebral bodies which are no denser than their disks are severely porotic. On this basis the data confirm our previous suggestion (Barnett and Nordin, 1960, 1961) that our original scoring procedure tends to underdiagnose osteoporosis-that is, it produces false negatives but no false positives. It would in fact appear from Fig. 7 that vertebral biconcavity develops at that stage in osteoporosis when the vertebral bodies have about the same radiographic density as the disks. It may seem surprising that the vertebral bodies can even become less dense than the disks, but we attribute this to the replacement of bony trabeculae by marrow fat.

Our results appear to resolve the problem of the difference between spinal and peripheral osteoporosis. We had previously suggested (Nordin, 1961 ; Barnett and Nordin, 1961) that peripheral osteoporosis might be a late stage in a disease process initiated in trabecular bone, particularly that of the spine, and had pointed out that the occurrence of pure peripheral osteoporosis might be an artifact of our original technique in that it failed to detect early spinal osteoporosis. This now appears to be the case. Examination of Fig. 6 shows that all our cases of peripheral osteoporosis with one exception have low R.V.D. ratios, and we are therefore satisfied that although spinal (that is, trabecular) osteoporosis may occur without peripheral (that is, cortical) osteoporosis, the reverse does not occur. In generalized osteoporosis the first loss of bone is trabecular and only later does cortical loss occur. This accords, with the experimental evidence of the effect of negative calcium balance in animals (Nordin, 1960), and is therefore compatible with the concept that clinical osteoporosis could be due to prolonged negative calcium balance.

\section{Summary}

A method is described for assessing the density of the lumbar vertebrae by comparison with the intervertebral disks. This procedure is designed to eliminate the effect of soft-tissue variation.

A lateral tomogram is obtained through the centre of the lumbar spine and of a dissected specimen of lumbar spine mounted in formol-saline and placed next to the subject.

The film images of the spine and standard are fed through a Laurence-Locarte recording densitometer and the difference between the average density of the disks and the average density of the bodies is determined. This difference in the spine is divided by the same difference in the standard, and the ratio is called the relative vertebral density.

The results obtained are compared with the $x$-ray scores of Barnett and Nordin. This comparison suggests that vertebral biconcavity develops at the stage when the vertebral body is of about the same density as the intervertebral disk.

In severe osteoporosis the vertebral bodies are less dense than the intervertebral disks.

This work was carried out during the tenure of grants from the Medical Research Council, International Atomic Energy Agency, and Glaxo Laboratories.
REFERENCES

Barnett, E., and Nordin, B. E. C. (1960). Clin. Radiol., 11, 166. - (1961). Brit. J. Radiol., 34, 683.

Bywaters, E. C. L. (1948). Clin. Sci., 6, 281.

Doyle, F. H. (1961). Brit. J. Radiol., 34, 698.

Keane, B. E., Spiegler, G., and Davis, R. (1959). Ibid., 32, 162.

Koch, W., and Kaplan, D. (1961). Lancet, 1, 377.

Mayo, K. M. (1961). Brit. J. Radiol., 34, 693.

Nordin, B. E C. (1960), Clin. Orthop., 17, 235.

(1961). Lancet, 1, 1011.

\section{METABOLISM OF ${ }^{59} \mathrm{Fe}-\mathrm{SORBITOL}$ COMPLEX IN MAN}

BY

\section{G. WETHERLEY-MEIN, M.D.}

J. G. BUChaNAN, M.B., Ch.B. U. H. GLASS, F.I.M.L.T.

AND

\section{C. PEARCE, A.R.T. (Canada)}

Louis Jenner Laboratory, St. Thomas's Hospital and Medical School, London

Recent reports of the satisfactory response of irondeficient patients to intramuscular administration of an iron-sorbitol-citric-acid complex (iron-sorbitol) (Andersson, 1961 ; Swedberg, 1962) suggested an investigation in man of the injection-site clearance, organ distribution, and utilization for haemoglobin synthesis of this complex labelled with ${ }^{59} \mathrm{Fe}$. The pattern of the investigation reported here is similar to the investigation of ${ }^{59} \mathrm{Fe}$ labelled iron dextran previously carried out in this department (Grimes and Hutt, 1957).

\section{Materials and Subjects}

${ }^{59} \mathrm{Fe}$-labelled Iron-sorbitol. - The physical and chemical properties of stable iron-sorbitol are described elsewhere (Lindvall and Andersson, 1961 ; Svärd, 1961 ; Svärd and Lindvall, 1961). The material used in the present study was prepared on a laboratory scale by standard manufacturing technique to contain $50 \mathrm{mg}$. of iron and about $10 \mu \mathrm{c}$. of ${ }^{59} \mathrm{Fe}$ per $\mathrm{ml}$. The labelled material was checked by electrophoresis and gel-diffusion technique and demonstrated identical properties with the commercially available product " jectofer." The preparation was made available in 2-ml. ampoules.

Subjects.-Ten patients were studied. The clinical diagnosis, age, and initial haematological findings are shown in the Table. Cases 1, 2, and 3 were regarded as haematologically normal controls, although, in retrospect, Case 2 may have been marginally iron-deficient. The remaining seven patients had varying degrees of untreated iron deficiency. All were, by selection, in the fourth decade or older.

\section{Methods}

Administration and Dosage.-Eight of the patients were given $1.5-1.6 \mathrm{ml}$. of labelled iron-sorbitol intramuscularly (75 mg. iron: $10 \pm 2 \mu \mathrm{c}$. ${ }^{59} \mathrm{Fe}$ ). In Case 1 the use of the buttock as injection site invalidated surface counting over the sacrum, and in the remaining seven patients (Cases 2, 3, 4, 7, 8, 9, and 10) the deltoid site was used. The two patients (Cases 5 and 6) in whom the iron was given intravenously received only $0.5 \mathrm{ml}$. of iron-sorbitol ( $25 \mathrm{mg}$. iron : $3-4 \mu \mathrm{c} .{ }^{59} \mathrm{Fe}$ ), since 